\title{
Is There a Relationship Between Disease Control and Liver Function in Children with JIA Receiving Treatment with Methotrexate?
}

\section{Kathryn Harrison and Satyapal Rangaraj}

Nottingham Children's Hospital NHS Trust, Nottingham, United Kingdom

*Corresponding author: Satyapal Rangaraj, Consultant in Paediatric and Adolescent Rheumatology E Floor, East Block, Nottingham Children's Hospital, Nottingham University Hospital NHS Trust Nottingham NG7 2UH, United Kingdom, Tel: 0115 9249924, Ext: 64493; E-mail: Satyapal.Rangaraj@nuh.nhs.uk

Rec date: October 01, 2015; Acc date: October 24, 2015; Pub date: November 03, 2015

Copyright: $\odot 2015$ Harrison K. This is an open-access article distributed under the terms of the Creative Commons Attribution License, which permits unrestricted use distribution, and reproduction in any medium, provided the original author and source are credited.

\begin{abstract}
Juvenile Idiopathic Arthritis (JIA) is one of the commonest chronic diseases of childhood. Despite significant advances in the field of biologics offering a much wider a range of treatment options, Methotrexate remains the 1st line disease modifying drug [1] especially for those with polyarticular disease or associated uveitis. Response to methotrexate is variable and though work is ongoing to try and predict those who will respond well, this has not yet translated into clinical practice. Methotrexate can be associated with significant side effects [2,3] and adherence can be challenging. Currently assessment of adherence in the patient with apparently resistant disease relies on patient or parent reporting, which may not always be accurate.
\end{abstract}

Liver function is routinely monitored in children and young people (CYP) on Methotrexate to look for evidence of liver toxicity [4-6]. It is not uncommon for transaminases to be abnormal while taking methotrexate [7]. A perceived relationship between normal liver function and methotrexate adherence has been reported by some practitioners [8].

Keywords Juvenile idiopathic arthritis; Methotrexate; Liver; Rheumatology; Physiotherapy

\section{Aim}

Our aim was to evaluate the relationship between liver function and disease control in CYP with JIA being treated with methotrexate. Specifically, we wanted to evaluate whether those with "low-normal" liver function had evidence of poorer disease control.

\section{Methods}

All CYP aged 0-18 currently being treated with methotrexate by the Rheumatology Team at Nottingham Children's Hospital $(\mathrm{NCH})$ were identified via the nursing database. CYP with a non-Juvenile Idiopathic Arthritis diagnosis or systemic JIA were excluded. At $\mathrm{NCH}$ blood monitoring tests are taken at the time of clinic appointments, so therefore we anticipated that most blood test results could be linked to a corresponding episode of clinical care. Also all monitoring blood tests take place at the hospital and therefore blood tests results would be available on the hospital computer system. The study did not include CYP whose care was being delivered by outreach services as their methotrexate monitoring is managed by their local team.

All ALT results available for eligible patients were identified from the hospital computer blood results system from 1/11/2013 to 31/10/2014. ALT results are reported as Units/Litre and were categorised into $\leq 10,11-15,16-45$ and $>45$. These categories were chosen because we specifically wanted to look at the relationship between disease control and "low-normal" liver function. Due to the low number of ALT results $\leq 10$, clinical data was collected for corresponding episodes of clinical care for all of these results. For all the other categories of ALT a random number generator was used to select those results for which further data would be collected. Clinical data was collected retrospectively from clinical records; clinical episodes within 2 weeks of the blood test were used to correspond with an ALT result. Clinical data was extracted from computerized hospital letters, patient notes and physiotherapy records. Data was collected for all of the variables included in the JADAS score and documentation of adherence to methotrexate therapy.

\section{Results}

There were 20 episodes of ALT $\leq 10$ in 15 different patients, 71 ALTs 11-15 in 33 patients, $14616-45$ in 70 patients $75>45$ in 34 patients. Data was collected for 20 episodes $\leq 10,21$ of $10-15,23$ of $16-45$ and 24 of $>45$. Due to the retrospective nature of the data collection there was insufficient data available to analyze further the full components of the JADAS score, so hereafter we only looked at the active joint count and ESR. Once hospital records were looked at 3 further exclusions were made (Table 1).

\begin{tabular}{|l|l|l|l|l|}
\hline ALT & $\leq 10$ & $11-15$ & $16-45$ & $>45$ \\
\hline Number of results & 20 & 21 & 23 & 24 \\
\hline Number of patients & 15 & 19 & 19 & 20 \\
\hline Further exclusions & $\begin{array}{l}\text { (Not yet started } \\
\text { MTX) }\end{array}$ & 0 & 1 (JDM overlap) & 0 \\
\hline $\begin{array}{l}\text { Analysed results/ } \\
\text { number of pt }\end{array}$ & $18 / 14$ & $21 / 19$ & $22 / 18$ & $24 / 20$ \\
\hline $\begin{array}{l}\text { Active joint count } \\
\text { documented }\end{array}$ & 14 & 16 & 20 & 22 \\
\hline ESR available & 14 & 20 & 24 & 23 \\
\hline
\end{tabular}

Table 1: Baseline data. 


\section{ESR}

A trend towards a lower ESR was noted in those episodes of care corresponding to higher ALTs, but this did not reach statistical significance (Figure 1).

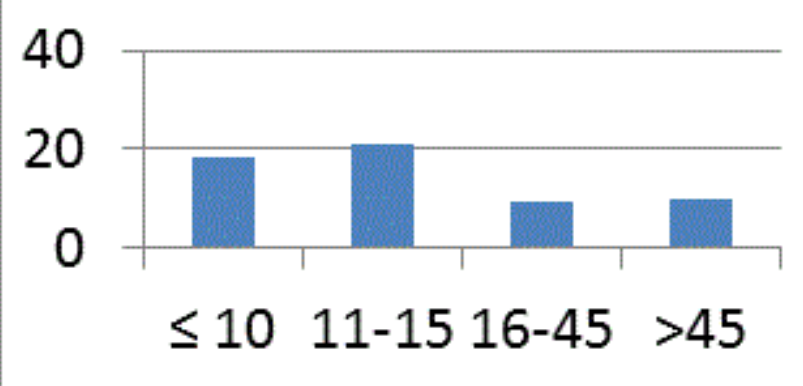

Figure 1: Trend towards a lower ESR.

When the groups were combined into ALT $\leq 15$ and ALT $>15$ then a significant difference was seen between the 2 groups in terms of ESR $(\mathrm{p}=0.001)$ using the unpaired students T-test (Figure 2).

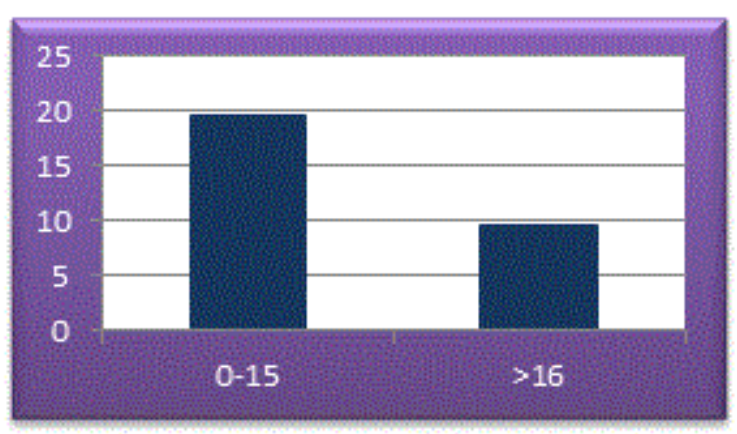

Figure 2: Average joint count.

The active joint count in those episodes of care associated with ALT $>45$ was lower than those with lower ALTs (Figure 3).

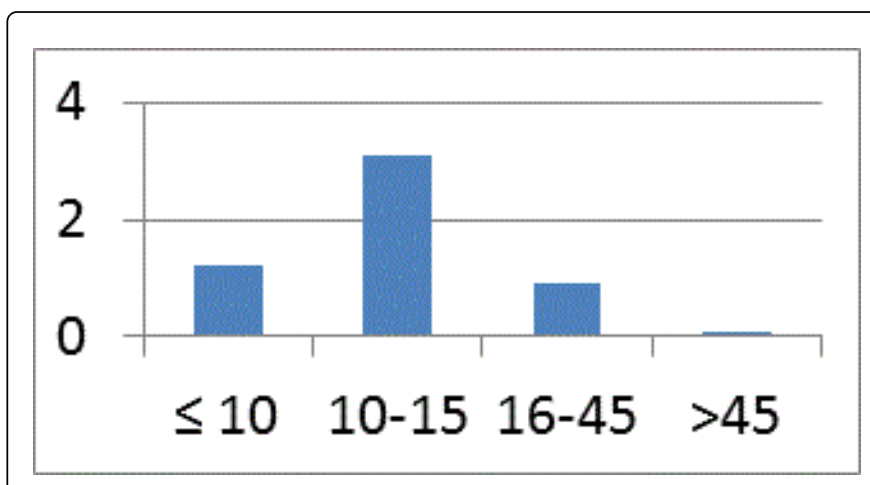

Figure 3: Active joint count.

Once combined the trend was clearer and reached statistical significance using the unpaired students $\mathrm{t}$-test $(\mathrm{p}=0.0174)$ (Figure 4).

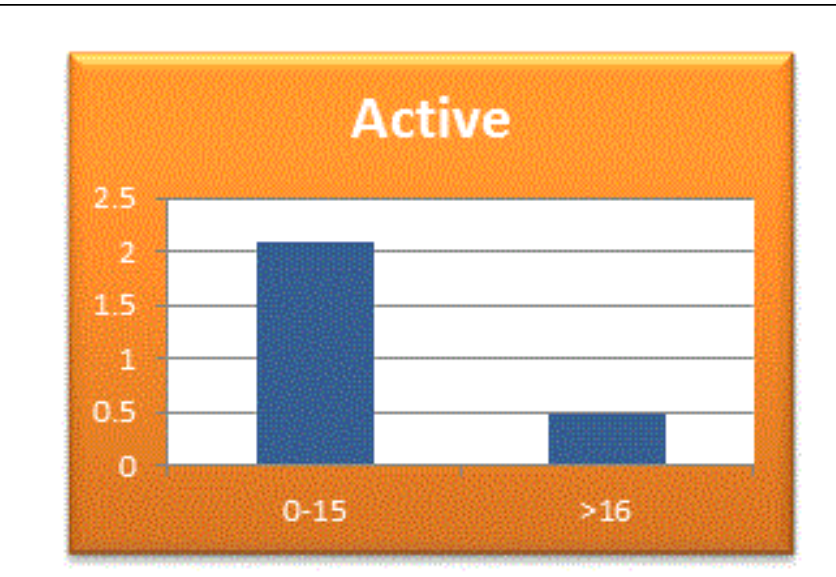

Figure 4: Statistical significance.

\section{Conclusions}

This is a small study. The data was retrospectively collected and consequently data was insufficient to analyze against validated composite measures of disease control. We did not look at other markers which could influence disease activity or the hepatic effects of methotrexate, such as use of additional drugs. It is likely that a number of patients will have been taking Non-Steroidal Anti-Inflammatory Drugs (NSAIDs), specifically Ibuprofen. However, given the apparent increase in the disease activity level in those with "low-normal" ALT, we would anticipate higher use of NSAIDs in this group for symptomatic relief, so we feel it is unlikely to introduce a bias into the results. This would however require further exploration in more detailed studies. It should also be noted, that although NSAIDs have hepatic dysfunction as a recognized side-effect, no association has been made between NSAID use and hepatotoxicity on methotrexate in JIA.

Despite some limitations, we believe this data does demonstrate a potentially interesting phenomenon in a significant difference in disease control between those with "low normal" ALTs and those with "high normal or abnormal" ALTs in 2 of the components of the JADAS score. This effect is observed despite the fact that those with abnormal LFTs are more likely to have had deliberate missed doses of methotrexate due to abnormal liver function, or intercurrent infections causing abnormal liver function.

Previous studies have reported variations in liver toxicity with different genetic polymorphisms involved in methotrexate metabolism, and in cellular methotrexate polyglutamate patterns $[9,10]$. These have exciting potential to help us predict who is likely to develop toxicity related to methotrexate and to tailor treatment to individual genetic makeup. Polymorphisms which may predict disease control have not correlated with those which may predict toxicity, and the same is true for those studies looking at polyglutamate patterns $[9,10]$. This project aimed to ask a slightly different question having looked specifically to see if those with "low-normal" liver function on methotrexate have poorer disease control. We believe this is the first study to report such an association. There was insufficient data to assess drug adherence and it is impossible to say from the data whether the observed effect is a consequence of adherence or individual variation in metabolism of methotrexate. If this result is a function of poor adherence rather than 
Citation: Harrison K, Rangaraj S (2015) Is There a Relationship Between Disease Control and Liver Function in Children with JIA Receiving Treatment with Methotrexate?. J Arthritis 4: 173. doi:10.4172/2167-7921.1000173

Page 3 of 3

individual response to methotrexate, then this may go some way to explaining the lack of association between hepatic toxicity and efficacy in previous studies. We would not advocate the use of ALT as a marker of response to treatment or adherence based purely upon this data. This does however potentially represent a cheap and readily available method of assessing either adherence, or the individual response to methotrexate and therefore warrants further investigation in a more robust study.

\section{References}

1. http://www.bspar.org.uk/pages/clinical_guidelines.asp

2. Gutierrez-Ureña S, Molina JF, García CO, Cuéllar ML, Espinoza LR (1996) Pancytopenia secondary to methotrexate therapy in rheumatoid arthritis. Arthritis Rheum 39: 272-276.

3. Lateef O, Shakoor N, Balk RA (2005) Methotrexate pulmonary toxicity. Expert Opin Drug Saf 4: 723-730.

4. Kremer JM, Kaye GI, Kaye NW, Ishak KG, Axiotis CA (1995) Light and electron microscopic analysis of sequential liver biopsy samples from rheumatoid arthritis patients receiving long-term methotrexate therapy. Followup over long treatment intervals and correlation with clinical and laboratory variables. Arthritis Rheum 38: 1194-1203.
5. Kremer JM, Furst DE, Weinblatt ME, Blotner SD (1996) Significant changes in serum AST across hepatic histological biopsy grades: prospective analysis of 3 cohorts receiving methotrexate therapy for rheumatoid arthritis. J Rheumatol 23: 459-461.

6. Kremer JM, Alarcón GS, Lightfoot RW Jr, Willkens RF, Furst DE, et al. (1994) Methotrexate for rheumatoid arthritis. Suggested guidelines for monitoring liver toxicity. American College of Rheumatology. Arthritis Rheum 37: 316-328.

7. Salliot C, van der Heijde D (2009) Long-term safety of methotrexate monotherapy in patients with rheumatoid arthritis: a systematic literature research. Ann Rheum Dis 68: 1100-1104.

8. Hawley DP, Camina N, Rangaraj S (2011) British isles survey of methotrexate monitoring practice during treatment of juvenile idiopathic arthritis. Semin Arthritis Rheum 2011 40: 358-64.e1-2.

9. Becker ML, Gaedigk R, van Haandel L, Thomas B, Lasky A, et al. (2011) The effect of genotype on methotrexate polyglutamate variability in juvenile idiopathic arthritis and association with drug response. Arthritis Rheum 63: 276-285.

10. van Dijkhuizen EH, Wulffraat NM (2014) Prediction of methotrexate efficacy and adverse events in patients with juvenile idiopathic arthritis: a systematic literature review. Pediatr Rheumatol Online J 12: 51. 